\title{
Modelling the ISM and Star Formation in Galaxy Formation Simulations
}

\author{
P R Williams \\ Shanghai Astronomical Observatory, Chinese Academy of Sciences, 80 \\ Nandan Road, Shanghai 200030, PR China
}

\section{A $\mathrm{H}$ Nelson \\ Department of Physics and Astronomy, Cardiff University, Cardiff CF2 3YB, Wales, UK}

\begin{abstract}
Using a set of 60 Tree-SPH simulations of the formation of an individual galaxy, including star formation physics, we quantitatively assess the numerical accuracy and convergence of these methods. For simulations using 24000 particles in total, the gross structures of the resultant galaxies are reliably resolved.
\end{abstract}

Treecode gravity and Smoothed Particle Hydrodynamics (SPH) are commonly used in galaxy simulations. But due to the high computational cost of such simulations, and the large number of physical and numerical parameters which specify any given simulation, it has proved difficult to quantitatively assess the veracity of solutions in a galaxy formation context. Simple test cases for which analytic or semi-analytic solutions are known, such as the Plummer sphere or the Riemann shock tube, are indispensable. However, their weakness is that they do not provide a test of all of the physical processes at play in a galaxy formation simulation simultaneously.

In order to quantitatively assess the reliability of the Treecode-SPH methods in galaxy formation simulations, we have adopted idealised galaxy formation initial conditions which produce a grand-design disk galaxy similar in properties to the Milky Way (Williams \& Nelson, 2001), and re-run these initial conditions more than 60 times, systematically varying all numerical parameters. The numerical parameters which specify a simulation (with the values used in a reference simulation given in parenthesis) are: number of gas particles (8146), number of dark matter particles (8219), number of star particles formed if all gas mass is converted into stars (8000), gravitational softening length $(1.75 \mathrm{kpc})$, treecode cell opening angle criteria (0.7), inclusion of quadrupole terms in the calculation of gravitational forces (included), number of gas particles contained within $2 h(50)$, artificial viscosity parameters $\alpha$ and $\beta(1,2)$, the number of star particles spawned in each star particle creation event (8, see Williams (1998) for details of the star formation algorithm), and a Courant number (0.1). In addition, there are a number of purely numerical variables and factors: the compiler and compiler options, processor model, the number of processors used (the code is written for use on a distributed parallel computer), and random number generator seeds (used in the star formation algorithm). 

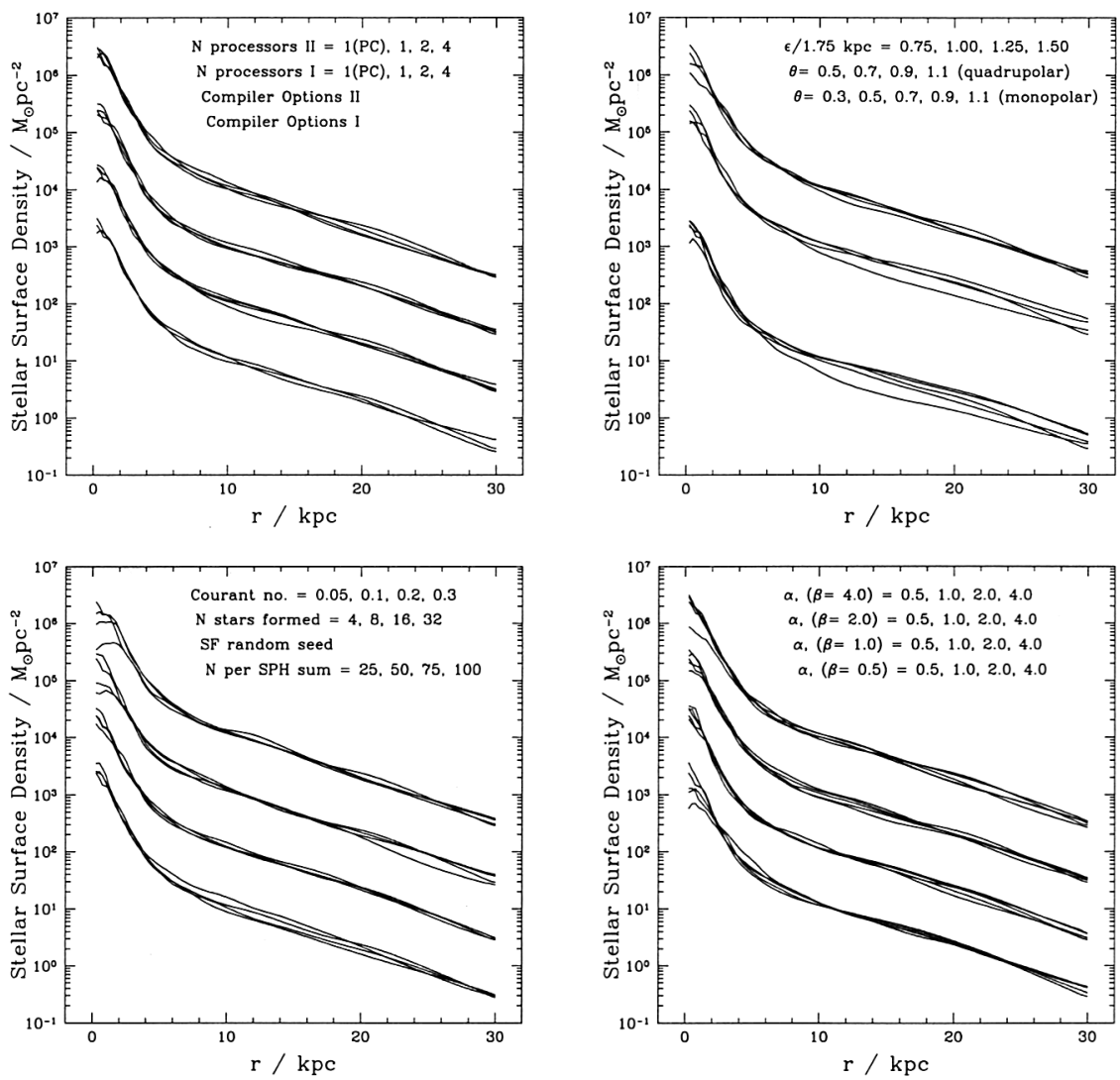

Figure 1. Variations in the stellar mass surface density profile at $t=12$ Gyr. A reference simulation is shown with a bold line, and is common to all plots. The sets of simulations are offset from one another in order to facilitate comparison, the lowest set having zero offset ( $\mathrm{N}$ processors I and II are different parallel algorithms).

In summary, the simulations have shown that while the evolution of component energies are sensitive to specific numerical parameters at the $20 \%$ level (particularly to the gravitational softening length, and to the gravitational force accuracy); this has no effect on the gross structure of the resultant numerical galaxy, which is reliably resolved, see Figure 1.

\section{References}

Williams, P. R., 1998, PhD Thesis, Cardiff University

Williams, P. R., Nelson A. H., 2001, A\&A, 374, 839 